\title{
INFECCIÓN POR Enterobius vermicularis EN PREESCOLARES DE COMUNIDADES RURALES
}

\section{INFECTION FOR Enterobius vermicularis IN KINDERGARTEN CHILDREN OF IRURAL COMMUNITIES}

\author{
Rodolfo Talledo y Julia Castro*
}

\section{RESUMEN}

Para validar la influencia que tiene la infección por Enterobius vermicularis en el rendimiento escolar, se tomó una muestra de 123 niños cuyas edades oscilaban de 3 a 6 años y que constituían toda la población escolar de 6 Centros de Educación Inicial, ubicados en las comunidades rurales de Carabayllo y Santa Rosa de Quives, en Lima, Perú. El estudio comprendió, además del examen parasitológico, la determinación del estado nutricional de los niños, utilizando la técnica de Graham modificada y el índice de KanawatiMc Laren, respectivamente.

Se halló 34,14\% de niños parasitados con Enterobius vermicularis y el $18,08 \%$ con desnutrición proteico-calórica leve. La correlación de los resultados por medio del análisis de varianza nos permitió concluir, que la infección por Enterobius vermicularis en los preescolares no influye en el rendimiento escolar, aunque no podemos generalizar este resultado, porque no se evaluaron otros enteroparásitos y además el aprestamiento inicial es básicamente motor antes que intelectual. La malnutrición afectaría a los nin̄os de 5 años y no asi a los de 3 y 4 años, aunque el método utilizado no fue el más adecuado.

Palabras clave: Enterobius vermicularis, preescolares, índice de Kanawati-Mc Laren, método de Graham.

\begin{abstract}
A group of 123 children aged 3 to 6 was chosen to study the effects that Enterobius vermicularis infection has on the school children's educational output. These children were the whole pre-scholar population of the kindergarten rural community in Carabayllo and Santa Rosa de Quives in Lima, Perú. This study included a parasitologic and nutritional examination of the children, using the modified Graham's techniques and the nutritional method of Kanawati-Mc Laren.

The result was 34,14 per cent of children with Enterobius vermicularis, and 18,08 per cent with a light protein - caloric malnutrition. The relation between the results from the varianza analysis enables us to conclude that Enterobius vermicularis infection does not have an effect on the children's performance in the school; however we can not generalize on this result because no other intestinal parasites were examined and the initial development is more motional than intelectual. Malnutrition was present in children of 5 years old and not in those of 3 and 4 years old who go to school according to our pre-scholar system and the method was not adequate.
\end{abstract}

Key words: Enterobius vermicularis, kindergarten children, method of Kanawati - Mc Laren, method of Graham.

\section{INTRODUCCIÓN}

En la mayoría de las poblaciones escolares se presentan deficientes condiciones de vida y de alimentación, y se justifican las afirmaciones de que estos factores influyen en el bajo rendimiento escolar, especialmente en

Fac. Ciencias Biológicas. Laboratorio de Inmunologia Parasitaria y Epidemiología. UNMSM. E-mail:d190007@unmsm.edu.pe. aquellas ubicadas en zonas urbano-marginales (Bedriñana, 1987; Matta, 1984).

La Organización Panamericana de la Salud ha reportado para paises de América Latina una elevada tasa de parasitosis intestinal y considera que la principal causa de ello es la contaminación del suelo con heces humanas, además de la carencia de una adecuada infraestructura sanitaria y el desconocimiento de normas sanitarias (Botero, 1981); en estas condiciones, los más afectados son los niños. 
Mediante el método de Graham, se examinaron 129 niños del Pueblo Joven Israel de Arequipa, y se obtuvo $42,6 \%$ de parasitismo por Enterobius vermicularis; los más afectados fueron aquellos cuyo intervalo de edad estuvo entre los 5 y 10 años (Castro, 1984). En Comunidades rurales de ChosicaHuarochiri se evaluaron 348 niños comprendidos entre los 6 meses y 5 años de edad, y se halló $86,78 \%$ de infección por enteroparásitos, y de ellos Enterobius vermicularis presentó la más alta prevalencia $(48,5 \%$ ) (Castro et al. 1991).

La prevalencia de Enterobiosis en escolares de un área urbano-marginal de Arequipa reportó $56,56 \%$ de parasitismo y el índice de hacinamiento de 1,1 (58,26\%) (Delgado, 1995). A través de un estudio prospectivo longitudinal se investigó la Enterobiosis en 386 niños de 2 a 12 años de Trujillo, y se encontró $44,47 \%$ de positividad; el grupo etario más afectado estuvo entre 6 y 8 años (Mejía,. 1995).

El mantenimiento de la Enterobiosis en la población infantíl se ve favorecido por los altos índices de hacinamiento ( 2,2 ) de los pobladores en las viviendas; debido al número promedio de personas por habitación y por número de camas (Castro et al. 1998 ).

Los niños infectados por enteroparásitos presentan dificultades nutricionales; es decir, existe una evidente interacción entre nutrición e infección, y se ha establecido que la infección deteriora la nutrición y que en el organismo desnutrido la infección se hace sinérgica, con efectos graves, prolongados y hasta letales. Esta deficiente disposición de nutrientes no provee al organismo del suficiente material para reposición corporal y obtención de la energía requerida, y afecta seriamente las actividades físicas y funcionales del organismo (Salomons y Rosales, 1986).

Las parasitosis intestinales, la mala nutrición, los diferentes estilos de vida y el saneamiento ambiental inadecuado, especialmente en poblaciones rurales y urbano-marginales, traen como consecuencia el retardo en el crecimiento, en la reposición tisular, en la actividad física y las condiciones fisiológicas especiales como la concentración, atención, disposición, etc. (Biolley y Gamboa, 1988). De este modo la actividad motora y cognoscitiva desarrolladas por el niño para el buen rendimiento escolar se hal lan seriamente disminuidas por el efecto de la interacción parasitismo-nutriciónsalubridad. (Botto et al. 1986).

El estudio permitió analizar aspectos epidemiológicos, nutricionales y de prevalencia de la Enterobiosis en la población preescolar considerada por Ango (1986) y Botero (1981) como el grupo etario de mayor vulnerabilidad.

El objetivo del presente trabajo fue evaluar la infección parasitaria causada por Enterobius vermicularis, y su probable influencia en el rendimiento escolar en la población infantíl de los Centros de Educación Inicial de las comunidades rurales de Carabayllo y Santa Rosa de Quives, ubicados en el Valle del río Chillón.

\section{MATERIAL Y MÉTODOS}

\section{ZONA DE ESTUDIO}

Se evaluaron 6 Centros de Educación Inicial (CEI) de la zona rural de los distritos de Carabayllo y Santa Rosa de Quives, del Valle de Chillón, ubicadas entre el km 25,5 y 48 de la Carretera a Canta, en ambas márgenes del río Chillón.

EI CEI N. 355 de la comunidad de Zapán; el CEI N. 367 de la comunidad de Macas y el CEI N. 864 de la comunidad de Trapiche, están ubicados en el distrito de Santa Rosa de Quives y los CEI N. 374 de la comunidad de Huatocay; el CEI N. ${ }^{\circ} 870$ de la comunidad de Punchauca y el CEI N. ${ }^{\circ} 876$ de la comunidad Río Seco, se encuentran en el distrito de Carabayllo. Las comunidades están alejadas de la carretera principal y el acceso fue a través de la carretera a Canta, por senderos es- 
trechos, pedregosos y con puentes peatonales improvisados.

En esta zona la población está dedicada a la crianza de ganado y a las labores agrícolas.

\section{TAMAÑO MUESTRAL}

Se evaluó la población total de los 6 Centros de Educación Inicial, de la zona rural de Carabayllo y Santa Rosa de Quives, que estuvo conformada por 123 niños en edad preescolar, cuyas edades oscilaron entre los 3 y 6 años. De ellos 25 niños tenían 3 años; 43 niños de 4 años y 55 niños de 5-6 años; siendo 57 de sexo masculino y 66 de sexo fermenino.

\section{METODOLOGÍA}

\section{ENCUESTA EPIDEMIOLÓGICA}

Antes de aplicar la encuesta se llevó a cabo la sensibilización de la población a través de etapas: la primera con las Directoras de los CEI de la jurisdicción de la USE 05 (Comas). La segunda etapa con las profesoras y además se visitaron e inspeccionaron los CEI. La tercera etapa se realizó con los padres de familia. En las comunidades de Macas y Punchauca se complementó con la visita domiciliaria.

Los datos de los niños se recogieron a través de una encuesta epidemiológica, para conocer los factores que favorecen la prevalencia de la Enterobiosis en la zona de estudio.También se aplicó una encuesta alimentaria-nutricional y parasitológica, y se estableció a partir de ella los hábitos alimentarios y los antecedentes del parasitismo de cada niño.

\section{MÉTODO PARASITOLÓGICO}

A cada niño se tomó en forma seriada 3 muestras de frotis anal, la primera mediante la técnica de Graham (Castillo et al. 1978) y las dos siguientes mediante la técnica de Graham modificada, que consistió en utilizar solo la cinta adhesiva, sin el portaobjeto.

\section{EVALUACIÓN NUTRICIONAL}

Para determinar el estado nutricional se aplicó la técnica de Kanawati-Mc Laren (Chevalier, 1993) que utiliza el índice braquiocefálico. Este índice se basa en mediciones del perímetro braquial y cefálico con ayuda de una cinta métrica inextensible. El valor de corte del método es de 0,290 ó 290; considera que hay malnutrición con valores debajo de este punto.

\section{RENDIMIENTO ESCOLAR}

La evaluación del rendimiento fue facilitada por las profesoras, y correspondió al primer semestre académico. Se evaluó motricidad, disposición y destreza manual, mientras que en la actividad cognoscitiva se evaluó la concentración y la captación. Los datos se recogieron en una ficha. Siendo las calificaciones en los CEI cualitativas y no habiendo una escala de equivalencias en el sistema vigesimal para cuantificar los resultados, le asignamos arbitrariamente valores numéricos y determinamos estadísticamente la correlación con el parasitismo y su estado nutricional.

Se estableció sólo dos categorías para los preescolares, la primera con rendimiento bueno/muy bueno y la segunda con rendimiento deficiente/regular.

\section{ANÁLISIS ESTADÍSTICO}

Se utilizó la Prueba de los Mínimos Cuadrados (MC) y el Análisis de Varianza (ANOVA), tomando como variables el resultado del examen parasitológico y el índice de malnutrición. El análisis de los resultados se realizó en forma desagregada según la edad.

El rendimiento escolar fue desagregado en cinco aspectos: concentración, captación, motricidad, disposición y destreza; se asignó el valor 1 para todo aspecto logrado y 0 para los no logrados. Se tomó la moda a estos valores y se recodificaron los resultados, generando nuevas variables. 


\section{RESULTADOS}

\section{CONDICIONES AMBIENTALES DE LAS COMUNIDADES}

Solo las comunidades de Huatocay y Trapiche poseen agua potable, y la población dispone de ella a través de pilones públicos, mientras las otras comunidades se abastecen de las acequias que se forman a partir del río Chillón, y la almacenan en baldes y bidones de plástico. La falta de servicios higiénicos y silos en las comunidades hace que los habitantes eliminen sus excretas a campo abierto. Asimismo, en ninguna comunidad se recoge la basura, por lo que se realiza la acumulación en basurales para luego incinerarlas (Figura 1).

Las viviendas presentaron construcciones rústicas $(96 \%)$ con una o dos habitaciones, paredes de adobe y esteras, suelo de tierra y techo de esteras o eternit. El 95\% de las viviendas tenía corrales en su perímetro (Figura 2). Las condiciones de la viviendas, los olores fétidos por la acumulación de excrementos de animales y la fermentación de los restos de alimentos permitieron observar la presencia de numerosos vectores mecánicos (moscas, mosquitos, cucarachas, etc.) (Figura 3).

\section{ENCUESTA ALIMENTARIA, NUTRI- CIONAL Y PARASITOLÓGICA}

Se encuestaron 123 niños, de los cuales el $31,87 \%$ manifestaron que se lavan las manos antes de comer; $21,8 \%$ se lava las manos después de defecar y el 12,19\% se baña diariamente. Todos los niños usaban calzado, pero se observó descuido en su aseo personal y además todos los niños habían recibido lactancia materna, aunque no precisaron el tiempo de ablactancia.

La población obtiene sus alimentos de los mercados, se abastece de hortalizas que ellos mismos cultivan y reciben donaciones a través de los CEI y de los Clubes de Madres. El $77,23 \%$ de las familias consumen carne una vez por semana. La dieta predominante en las familias es sopa de verduras $(85,3 \%)$, arroz con menestras $(78,8 \%)$, fideos $(78,8 \%)$, yuca o papa $(65,8 \%)$.

El examen clínico-nutricional reveló que el $26 \%$ de niños tenía el pelo decolorido y el $100 \%$ opaco y sin brillo. El 31,7\% presentó la conjuntiva pálida; el $8,1 \%$ con estomatitis angular en los labios y el $21,14 \%$ presentó caries; en todos los niños el aspecto de la lengua y de las encías fue normal. El 13,82\% manifestó dolores estomacales y diarreas; el 3,25\% refirió haber eliminado parásitos y el $6,3 \%$ haber recibido tratamiento.

TABLA 1. PREVALENCIA DE ENTEROBIOSIS EN PREESCOLARES DE LOS CEI DE LAS COMUINIDADES DE CARABAYLLO Y SANTA ROSA DE QUIVES

\begin{tabular}{|c|c|c|c|c|c|c|}
\hline \multirow{2}{*}{$\begin{array}{l}\text { COMUNIDADES } \\
\text { Punchauca }\end{array}$} & \multirow{2}{*}{$\begin{array}{l}\text { CEl } \\
870\end{array}$} & \multirow{2}{*}{$\begin{array}{c}\begin{array}{c}\text { CASOS } \\
\text { ESTUDIADOS }\end{array} \\
15\end{array}$} & \multicolumn{2}{|c|}{$\begin{array}{c}\text { CASOS } \\
\text { POSITIVOS }\end{array}$} & \multicolumn{2}{|c|}{$\begin{array}{c}\text { CASOS } \\
\text { NEGATIVOS }\end{array}$} \\
\hline & & & 4 & $26,66 \%$ & 11 & $73,34 \%$ \\
\hline Río Seco & 876 & 19 & 3 & $15,79 \%$ & 16 & $84,21 \%$ \\
\hline Huatocay & 374 & 16 & 5 & $31,25 \%$ & 11 & $68,75 \%$ \\
\hline Trapiche & 864 & 26 & 12 & $46,15 \%$ & 14 & $53,85 \%$ \\
\hline Zapán & 355 & 15 & 5 & $33,34 \%$ & 10 & $66,66 \%$ \\
\hline Macas & 367 & 32 & 13 & $40,62 \%$ & 19 & $59,38 \%$ \\
\hline TOTAL & & 123 & 42 & $34,15 \%$ & 81 & $65,85 \%$ \\
\hline
\end{tabular}




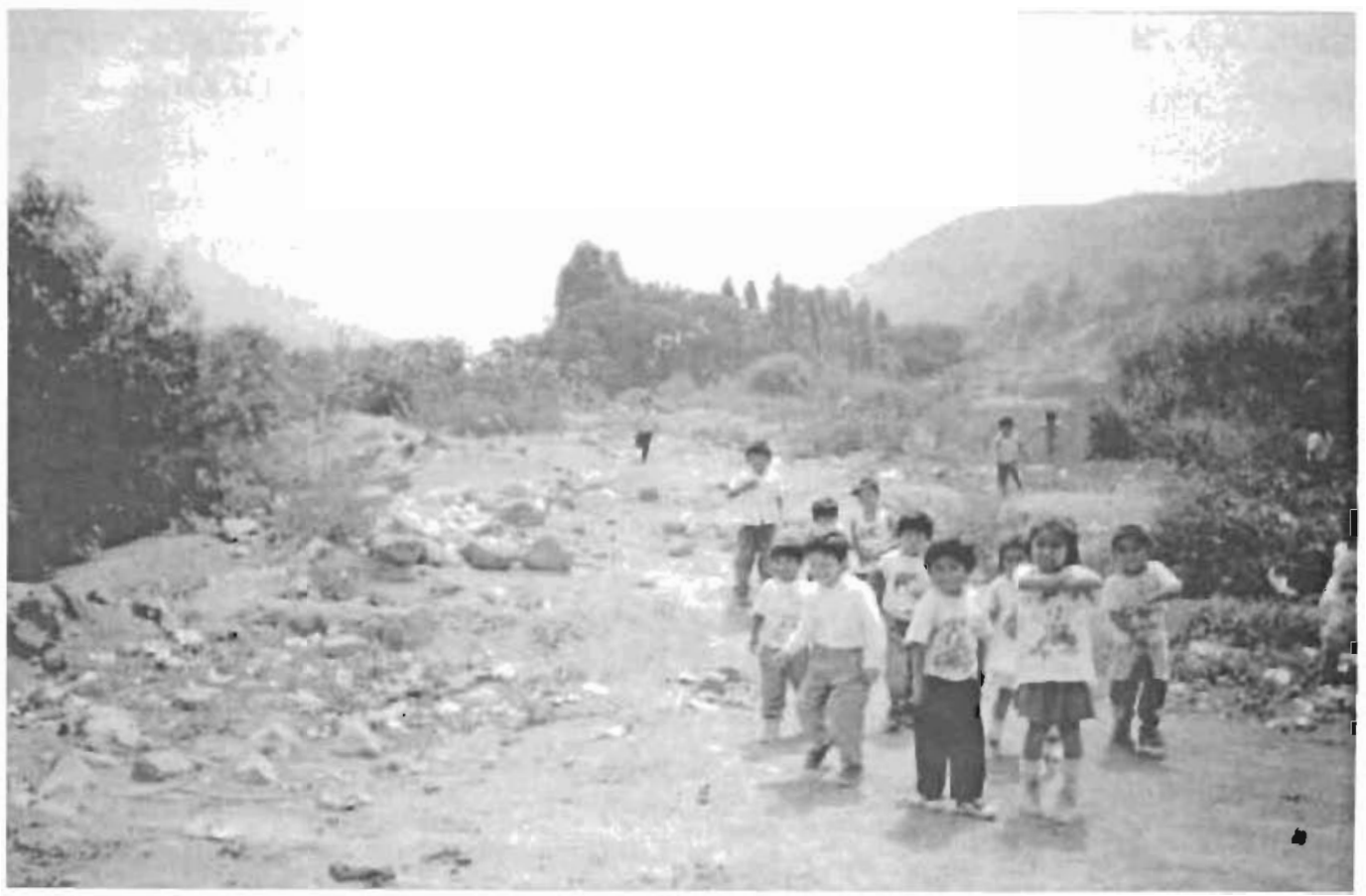

Figura 1. Acumulación de basura en el patio del Centro Educativo de Educación Inicial, donde los niños juegan.

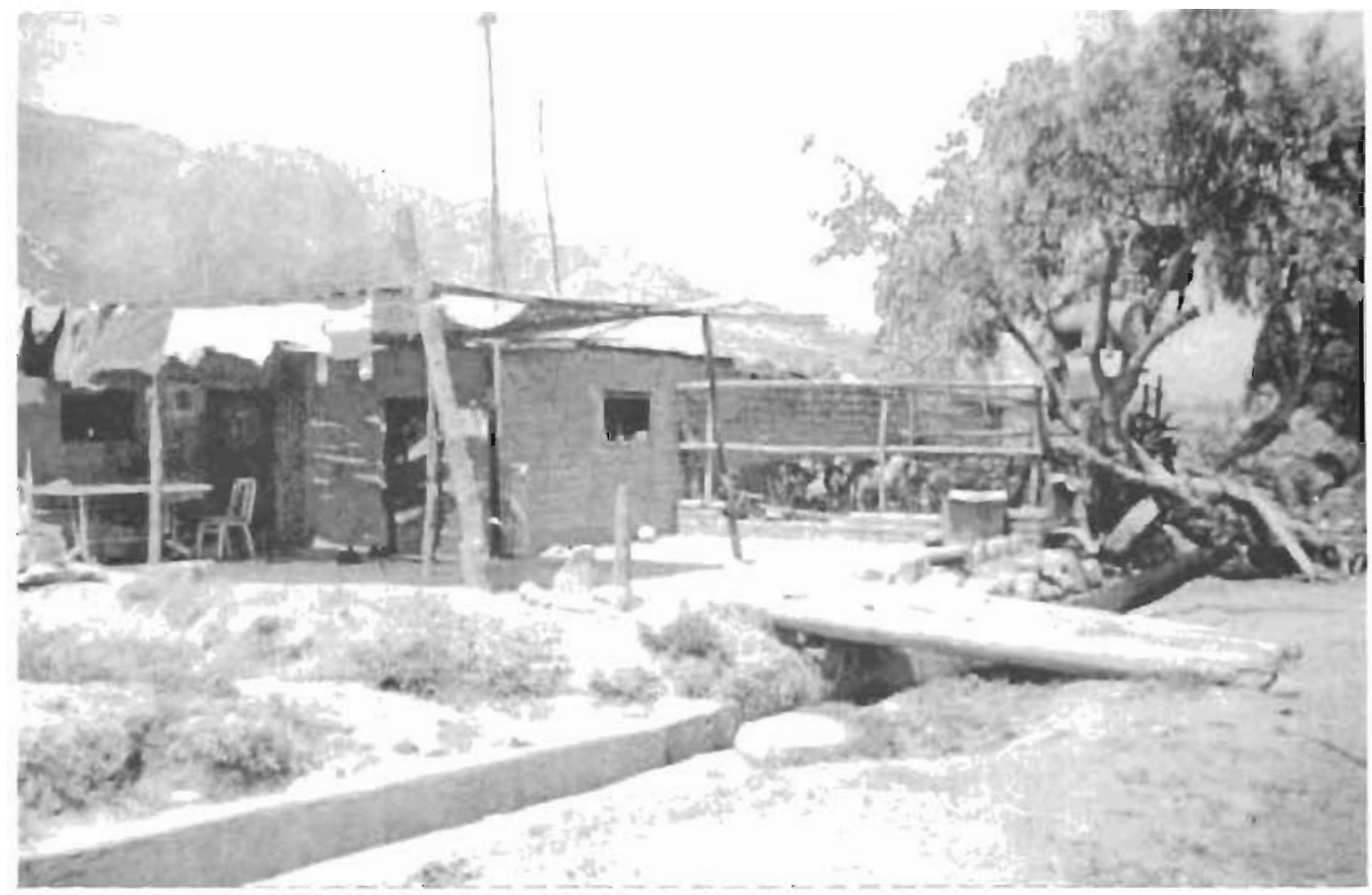

Figura 2. Viviendas de construcción rústica del Valle Chillón. 


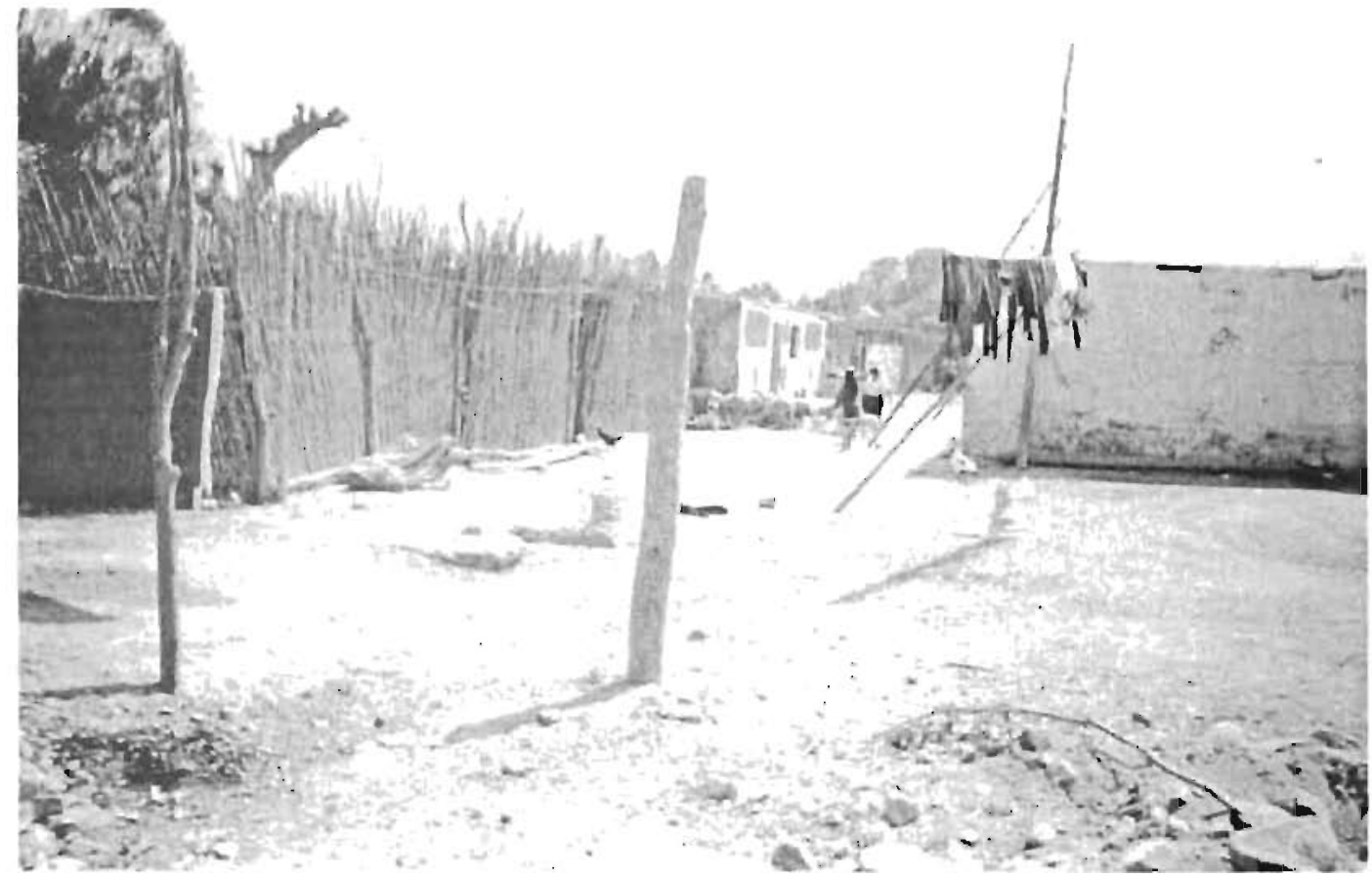

Figura 3. Presencia de corrales alrededor de las viviendas

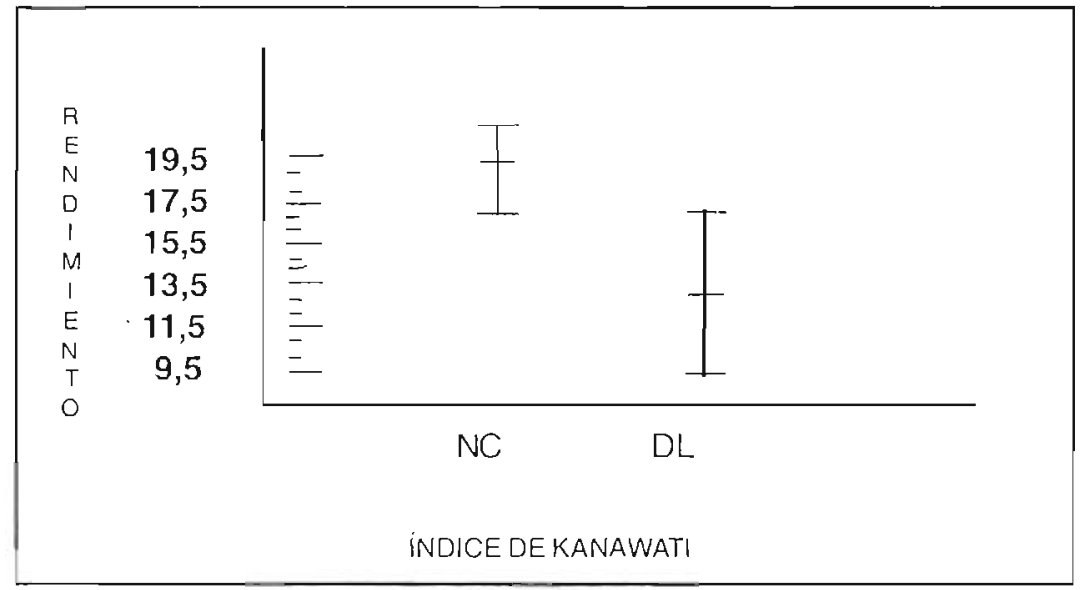

Figura 4. Comparación de los niveles del factor índice de Kanawati por rendimiento. NC: nutrición correcta, DL: desnutrición leve 
TABLA 2. PREVALENCIA DE ENTEROBIOSIS SEGUN SEXO Y EDAD DE LOS PREESCOLARES DE LAS COMUNIDADES DE CARABAYLLO Y SANTA ROSA DE QUIVES.

\begin{tabular}{|c|c|c|c|c|c|c|c|c|c|}
\hline \multirow{2}{*}{$\begin{array}{l}\text { EDAD } \\
(\text { Años })\end{array}$} & \multirow[t]{2}{*}{ CASOS } & \multicolumn{2}{|c|}{ POSITIVOS } & \multicolumn{2}{|c|}{ NEGATIVOS } & \multicolumn{2}{|c|}{ POSITIVOS } & \multicolumn{2}{|c|}{ NEGATIVOS } \\
\hline & & $\mathrm{N} .{ }^{\circ}$ & 90 & $\mathrm{~N}{ }^{\circ}$ & $\%$ & $\mathrm{~N} .{ }^{\circ}$ & $\%$ & $\mathrm{~N} .{ }^{\circ}$ & $\%$ \\
\hline 3 & 25 & 2 & 8,00 & 8 & 32,00 & 4 & 16,00 & 11 & 44,00 \\
\hline 4 & 43 & 9 & 20,93 & 14 & 32,56 & 5 & 11,63 & 15 & 34,88 \\
\hline $5-6$ & 55 & 10 & 18,18 & 14 & 25,45 & 12 & 21,81 & 19 & 34,54 \\
\hline TOTAL & 123 & 21 & 17.07 & 36 & 29,27 & 21 & 17,07 & 45 & 36,59 \\
\hline
\end{tabular}

TABLA 3. EVALUACIÓN NUTRICIONAL DE LOS PRE-ESCOLARES DE LOS CEI DEL VALLE CHILLÓN (Según el índice de Kanawati-Mc Laren)

\begin{tabular}{l|l|ll}
\hline UMBRAL & CONDICIÓN & \multicolumn{2}{|l}{ TOTAL } \\
NUTRICIONAL & & & \\
\hline $281-340$ & Desnur prot/calórica leve & 17 & $18,08 \%$ \\
$311-340$ & Estado nutricional correcto & 77 & $81,92 \%$ \\
\hline TOTAL & & 94 & \\
\hline
\end{tabular}

TABLA 4. INFLUENCIA DE LA ENTEROBIOSIS EN LA ACTIVIDAD COGNOSCITIVA DE LOS PREESCOLARES DE LOS CEI DEL VALLE CHILLÓN

\begin{tabular}{|c|c|c|c|c|c|c|c|c|c|c|c|c|c|c|}
\hline \multirow{2}{*}{$\begin{array}{l}\text { Rendim } \\
\text { Escolar }\end{array}$} & \multirow{2}{*}{$\begin{array}{c}\text { Casos } \\
\text { estud }\end{array}$} & \multirow{2}{*}{$\begin{array}{l}\text { Result Test } \\
\text { Graham }\end{array}$} & \multicolumn{2}{|c|}{ Punchauca } & \multicolumn{2}{|c|}{ RíoSeco } & \multicolumn{2}{|c|}{ Hualocay } & \multicolumn{2}{|c|}{ Trapiche } & \multicolumn{2}{|c|}{ Zapán } & \multicolumn{2}{|c|}{ Macas } \\
\hline & & & $\mathrm{N}^{\circ}$ & $\%$ & $\mathrm{~N}^{\circ}$ & $\%$ & $N^{\circ}$ & $\%$ & $\mathrm{~N}^{\circ}$ & $\%$ & $\mathrm{~N}^{\circ}$ & $\%$ & $N^{\circ}$ & $\%$ \\
\hline MUY & 8 & $\mathrm{P}(+)$ & 1 & 0,85 & - & - & - & - & . & - & - & - & 2 & 1,69 \\
\hline BUENO & & $N(-)$ & 2 & 1.69 & । & 0,85 & - & - & - & - & 1 & 0,85 & I & 0,85 \\
\hline \multirow[t]{2}{*}{ BUENO } & 57 & $P(+)$ & 2 & 1,69 & ! & 0,85 & 4 & 3,39 & 5 & 4,25 & 2 & 1,69 & 6 & 5,1 \\
\hline & & $N(-)$ & 4 & 3,39 & 8 & 6,8 & 7 & 5,95 & 5 & 4,25 & 3 & 2,55 & 10 & 8,5 \\
\hline \multirow[t]{2}{*}{ REGULAR } & 44 & $P(+)$ & - & - & 1 & 0,85 & I & 0,85 & 4 & 3,39 & 1 & 0,85 & 5 & 4,25 \\
\hline & & $N(-)$ & 4 & 3,39 & 6 & 5,1 & 4 & 3,39 & 7 & 5,95 & 3 & 2,55 & 8 & 6,8 \\
\hline \multirow[t]{3}{*}{ DEFIC. } & 9 & $P(+)$ & - & - & - & - & - & - & 3 & 2,55 & 1 & 0,85 & & - \\
\hline & & $N(-)$ & - & - & - & - & - & - & 2 & 1,69 & 3 & 2.55 & - & - \\
\hline & 118 & & 13 & & 17 & & 16 & & 26 & & 14 & & 32 & \\
\hline
\end{tabular}

\section{DIAGNÓSTICO PARASITOLÓGICO}

Utilizando el método de Graham modificado, se analizaron 123 muestras, y se observó $34,14 \%$ de Enterobiosis. No evaluamos la carga parasitaria, sin embargo observamos un elevado número de huevos por campo. El CEI N. 864 de la localidad de Trapiche presentó el más alto porcentaje $(46,62 \%)$, (Tabla N. $\left.{ }^{\circ} 1\right)$.

El análisis parasitológico evidenció que las niñas y los niños tienen igual prevalencia de parasitismo por Enterobius vermicularis $(17,07 \%)$; asimismo, se estableció que el grupo de 5-6 años presentó mayor número de casos positivos (Tabla N. ${ }^{\circ}$ ). 


\section{EVALUACIÓN NUTRICIONAL}

Por ausentismo de 29 niños a los CEI, solo se evaluaron 94 de ellos. El menor valor del perímetro braquial fue de $14 \mathrm{~cm}$ ( 3 niños) y el mayor valor encontrado fue de $19 \mathrm{~cm}$ (4 niños). El mayor valor obtenido para el perímetro cefálico fue de $54 \mathrm{~cm}$ y el menor $46 \mathrm{~cm}$. De acuerdo con el índice de Kanuwati el $18,08 \%$ presentó desnutrición proteica calórica leve y el $81,92 \%$ presentó un estado nutricional correcto (Tabla N. ${ }^{\circ}$ ).

\section{EVALUACIÓN DEL RENDIMIENTO ES. COLAR}

La evaluación cognoscitiva fue la siguiente: 57 niños con buen rendimiento, 44 con renclimiento regular, 8 con rendimiento muy bueno y 9 con rendimiento deficiente(Tabla $N{ }^{\circ} 4$ ). Mediante la evaluación motora se determinó que 15 niños tenían muy buena actividad motora, 65 presentaron buena motricidad, 36 con actividad regular y 2 con motricidad deficiente (Tabla $\mathrm{N}^{\circ}{ }^{5}$ ).

\section{ANALISIS ESTADÍSTICO}

Se analizaron de acuerdo a la edad, 94 niños, que tenían datos de la evaluación nutricional y del rendimiento escolar. Se procesaron 19 niños de 3 años de edad, y se obtuvo en la correlación rendimiento/parasitosis el nivel de significancia de 0,5977 , mientras que en la correlación rendimiento/malnutrición se obtuvo 0,8410 de significación.

La prueba de los MC permitió establecer que 14 de los niños tuvieron rendimiento de 15,41 , ligeramente debajo del promedio general ( 16,25$)$, aun cuando el resultado del examen parasitológico resultó negativo; contrariamente 4 niños con resultado parasitológico positivo presentaron como promedio 17,08 es decir, ligeramente superior al promedio general.

Se analizaron 30 niños de 4 años de edad. El nivel de significancia de la correlación rendimiento/parasitosis fue de 0,9269 y la correlación rendimiento/malnutrición 0,7382 . Veintidós niños sin parásitos y con nutrición co-

TABLA 5. INFLUENCIA DE LA ENTEROBIOSIS EN LA ACTIVIDAD MOTORA DE LOS PREESCOLARES DE LOS CEI DEL VALLE CHILLÓN

\begin{tabular}{|c|c|c|c|c|c|c|c|c|c|c|c|c|c|c|}
\hline \multirow{2}{*}{$\begin{array}{l}\text { Rendim } \\
\text { Motora }\end{array}$} & \multirow{2}{*}{$\begin{array}{l}\text { Casos } \\
\text { estud }\end{array}$} & \multirow{2}{*}{$\begin{array}{l}\text { Resuli. Tess } \\
\text { Grahann }\end{array}$} & \multicolumn{2}{|c|}{ Punchauca } & \multicolumn{2}{|c|}{ Ríoseco } & \multicolumn{2}{|c|}{ Huatocay } & \multicolumn{2}{|c|}{ Trapiche } & \multicolumn{2}{|c|}{ Zapán } & \multicolumn{2}{|c|}{ Macas } \\
\hline & & & N. ${ }^{\circ}$ & $\%$ & N. ${ }^{\circ}$ & $\%$ & $\mathrm{~N} .{ }^{\circ}$ & $\%$ & N. ${ }^{\circ}$ & $\%$ & $\mathrm{~N}{ }^{\circ}$ & $\%$ & $N^{\circ}{ }^{\circ}$ & $\%$ \\
\hline$M U Y$ & 8 & $P(+)$ & 1 & 0.85 & - & - & - & - & - & - & - & - & 3 & 2,55 \\
\hline BUENO & & $N(-)$ & 2 & 1,69 & 2 & 1.69 & - & - & . & - & 1 & 0.85 & 6 & 5,1 \\
\hline \multirow[t]{2}{*}{ BUENO } & 57 & $P(+)$ & 1 & 0,85 & I & 0,85 & 5 & 4,25 & 6 & 5,10 & 4 & 3,39 & 7 & 5,95 \\
\hline & & $N(-)$ & I & 0,85 & 5 & 4,25 & 9 & 7,65 & 13 & 11,05 & 7 & 5,95 & 6 & 5,1 \\
\hline \multirow[t]{2}{*}{ REGULAR } & 44 & $\mathrm{P}(+)$ & 1 & 0,85 & - & - & - & - & 6 & 5.10 & - & - & 3 & 2,44 \\
\hline & & $N(-)$ & 6 & 5.10 & 8 & 6,80 & 2 & 1.69 & 1 & 0,85 & 2 & 1.69 & 7 & 5.95 \\
\hline \multirow[t]{3}{*}{ DEFIC } & 9 & $P(+)$ & . & - & 1 & 0,85 & - & - & - & - & - & - & - & - \\
\hline & & $N(-)$ & I & 0.85 & - & - & - & - & - & - & - & - & - & . \\
\hline & 118 & & 13 & & 17 & & 16 & & 26 & & 14 & & 32 & \\
\hline
\end{tabular}


rrecta presentaron igual o mayor rendimiento respecto del promedio general.

La prueba de los MC determinó 16,97 como promedio en el rendimiento; 16,87 en 7 niños con resultados parasitológicos positivos; 17,07 en 22 niños con resultados negativos; 17,34 en 23 niños con nutrición correcta y 16,60 en 6 niños con desnutrición leve.

Se analizaron 44 niños de 5-6 años de edad. El nivel de significancia fue de 0,7155 para la correlación rendimiento/parasitosis y de 0,0151 para la correlación rendimiento/malnutrición. El promedio de rendimiento que presentó el grupo de 17 niños parasitados con $E$. vermicularis fue de 15,52 y el promedio de los 27 niños no parasitados fue de 15,03. Los niños con o sin parásitos mostraron rendimientos promedios equivalentes al promedio general.

La superioridad en el rendimiento se puede observar en aquellos niños sin desnutrición, respecto de los niños que acusaron desnutrición leve. Así 40 niños con nutrición normal tuvieron promedio de 18,06 y cuatro niños con desnutrición leve presentaron promedio de 12,05 (Figura 4).

\section{DISCUSIÓN}

Harrison (1988) sostiene que si bien la transmisibilidad de los oxiuros es permanente, es menor en ambientes cálidos, la baja frecuencia desafía cualquier explicación a este respecto. En nuestro estudio, se halló un alto porcentaje de viviendas con pisos de tierra, paredes de adobe-estera, que permiten que prolifere el polvo vehículo para la dispersión de los huevos de E. vermicularis, que es llevado por los pobladores fuera de sus viviendas. Teniendo en cuenta que los niños a partir de cierta edad realizan sus juegos y otras actividades fuera de casa, se justifica que los menores de 5 años presenten la mayor prevalencia de Enterobiosis, a diferencia de los de menor edad, quienes eran recogidos por sus padres y no jugaban en los mismos lugares.
El porcentaje de parasitismo por E. vermicularis $(34,14 \%)$ es menor al hallado por Castro et al. (1991) de 48,5\% en muestreos domiciliarios en comunidades urbano-marginales de Chosica-Huarochirí y a la encontrada por Contreras et al. 1993 (41,89\%). Los resultados obtenidos se explicarían por la dificultad para tomar las muestras a primera hora de la mañana, ya que los niños participan en las labores agrícolas y de pastoreo durante todo el año, junto con sus padres. Otra causa es la irregular asistencia de los niños a los CEI, o sino casi siempre llegan tarde, desaseados y sin haber ingerido alimento.

Sin embargo coincidimos con Benenson (1992), quien afirma que la Enterobiosis afecta todas las clases sociales y que la prevalencia es mayor en escolares, disminuye en preescolares y es casi nula en adultos.

La sensibilidad del método de Graham modificado no ha sido medida en el presente trabajo; sin embargo consideramos que su empleo no ha influenciado en los resultados.

En el Perú, un alto porcentaje de la población infantíl nace y crece en condiciones de privación psiconutricional de alto riesgo; de esto se conoce las consecuencias de la malnutrición severa, pero aún no se presta la debida atención a la malnutrición crónica, que es muy común en nuestra población (Majluf, 1993). Al correlacionar el estado nutricional con los datos clínicos y los hábitos alimentarios de la población, obtuvimos resultados que no reflejaron la realidad nutricional de los preescolares, pues durante las observaciones los niños presentaron para la misma edad una marcada diferencia en las características antropométricas, lo que habría provocado sesgo en los resultados. Según Latham (1991), los cambios pequeños en los límites antropométricos hacen suponer un estimado de variaciones grandes del orden de millones de niños que sufren malnutrición.

Los estudios de Castro et al.(1991) y Naupay et al. (1992) en comunidades rurales con geografía y características similares a las 
comunidades de Carabayllo y Santa Rosa de Quives revelaron mayor prevalencia de Enterobiosis que la encontrada en el presente estudio, porque emplearon otros indicadores nutricionales, por lo que deducimos que el indicador nutricional utilizado no fue el más adecuado.

Si bien el análisis estadístico determinó que el parasitismo por E. vermicularis no afectó significativamente el rendimiento de los preescolares; sin embargo éste sí fue afectado ligeramente por el estado nutricional, especialmente en los niños de mayor edad: Díaz, (1979) y Latham (1991) sostienen que los parásitos intestinales suelen producir anorexia, reducen la ingesta y absorción de alimentos, o producen la pérdida de ellos, lo que afecta el estado nutricional e indirectamente el rendimiento escolar. Celedón (1983) agrega otras variables que afectan el rendimiento escolar como las afectivas, personales y familiares; támbién sugiere que la desnutrición no actuaría sobre la función intelectual como una variable de tipo continuo. Esto podría ser la explicación para que no haya una marcada diferencia en el rendimiento escolar entre los niños con nutrición correcta y los que sufren malnutrición. También refiere que en los preescolares con malnutrición, las habilidades de orden abstracto se encuentran más afectadas que las manuales, sin embargo en los preescolares de mayor edad es entendible la influencia de la malnutrición en el rendimiento escolar.

Para determinar que la Enterobiosis y la malnutrición son la causa del rendimiento preescolar, se aplicó el ANOVA, y se obtuvo vaJores muy altos en el nivel de significancia en los niños de 3-4 años, por lo que afirmamos que la prueba no evidencia diferencias o que el rendimiento noes afectado por la malnutrición o el parasitisno por E. vermicularis. En los niños de 5 años, su rendimiento solo mostró diferencias significativas dependiendo del nivel nutricional, en forma similar a lo establecido por Soto et al. (1993). En terminos de parasitismo, no podernos hacer la misma afirma- ción, que podría deberse a las condiciones del muestreo, o bien por tratarse de una zona con temperatura cálida, coincidiendo con Harrison (1988) y Botero (1984).

\section{CONCLUSIONES}

1. Se delerminć que el $34,14 \%$ de los preescolares estuvieron parasitados por Enterobius vermicularis.

2. Las comunidades de los distritos de Carabaylio y Santa Rosa de Quives, ofrecen condiciones sanitarias y ambientales para la persistencia del parasitismo por Enterobius vermicularis.

3. El $18,08 \%$ do los preescolares presentó una leve malnutrición proteico calórica.

4. Se determinó que el rendimiento escolar solo es afectado por la desnutrición, según se observó en niños de 5 años.

\section{LITERATURA CITADA}

Ango, H.; Bedriñana. F. Huamán, G y Ortíz, G. 1986. Grado de parasilismo y relación de constantes hematométricas en niños en edad escolar en Ayacucho. Res. VIJl Cong. Nac. Biología. Alequipa I'crú.

Bedriñana, F. 1987. Oxiuriasis en pre-escolares y su relación con algunas variables epidemiológicas en Cangallo, Ayacucho. Res. VIII Cong. Nac. Biología. Arequipa-Perú.

Benenson, A. 1992. Ei control de las Enfermedades Transmisibles en el Hombre. Publicación Científica N. ${ }^{\circ} 538$ OPS, 617 pp.

Biolley, M. y Gamboa, C. 1998. Enteroparasitosis en una escuela de párvulos de la ciudad de Temuco IX Región Chile. Rev. Parasitología al Día 12:91-93.

Botcro, D. 1981. Persistencia de parasitosis intestinales endémicas en A mérica Latina. Bol. Oficina Sanitarid Panamericana (OPS) 90 (1):39-46

Botero, D. y Restrepo. M. 1984. Parasitosis Humanas. Corporación para Investigaciones Biológicas (CIB) Medellin, Colombia. 379 pp. 
Bolto, O ; Cabrera, A.; Segura, H., Perones, S.; Díaz, E.; Ramirez, L. y Carrasco, N. 1986. Sislema contínuo de intormación del estado nutricional: Ocl-Nov. 1984 y Julio 1985, en la zona de influencia del Hospital General Base Maria Auxiliadora. Rev. Diagnóstico 17(4):91-100.

Castillo, C.; Guillén, Z.: Cáceres, I., Náquira, C.: Tantaleán, M. y Ellioł, A. 1978. Manual de Mécodos de Diagnóstico en Parasitología Médica. UNMSM. 74 pp.

Castro, J. 1984. Enteroparasilosis en el Pueblo Joven Israel-Arequipa. Trabajo Exıramural Fac. Med. Humana UNMSM. Bol. Inst. Med. Trop. (1):35-38

Castro, J., García, E., Castro, E. y Mejia, A. 1991. Evaluación nutricional y prevalencia de parasitismo en comunidades urbano-marginales. Zona Alta. Rev. Per. Med. Trop. UNMSM (5):67-74.

Castro, J.; Huamán, M., Castro, E., Morales, L.y Nongrados, D. 1998. Evaluación del estado nutricional en escolares y aspectos epidemiológicos de la Oxyuriosis. Rev. Per. Parasitol. 13(1):36-41.

Celedón, J. 1983. Nutrición e inteligencia en el niño. Edit. Universidad de Santiago de Chile. 253 pp.

Contreras, O., Espinoza, I.; Albuquerque, M. y Solis, H. 1993. Prevalencia de parásitos intestinales en niños del A.A. H.H. Parque Porcino, Distrito de Ventanilla-Callao. Rev. Med. Trop. UNMSM $7(\mathrm{I}): 73-78$.

Chevalier, P. 1993. El índice de Kanawati-Mc Laren o relación brazo/cabeza: una técnica sencilla de conocer el estado nutricional de un niño. Rev. Diagnóstico 32: 25-27.

Delgado, M.; Lui, M. y Martínez, E. 1995. Prevalencia de Oxiuriasis en escolares de Arequipa. Res. II Cong. Per. Parasitología. pp.
Díaz, T. 1979. Investigación nutricional sobre la interferencia del parasitismo con diela de recuperación en niños que acuden a la posta médica de Huascala-Chosica. Tesis Bach. Med. Humana Univ. Federico Villarreal.

Harrison, R. 1988. Medicina Interna. Edit. Prensa Médica Mexicana. Univ. México 20 D. F. I: 1215 $\mathrm{pP}$

Latham, M. 1991. Malnutrición proteica-energélica. OPS. Instituto Internacional de Ciencias de la Vida (ILSI) Norteamérica. Copublicación científïca N. ${ }^{\circ} 532(5): 47-55$.

Matta, L. 1984. Interrelación entre desnutrición e infección. Separata de Nutrición N. $02-07$. Lima, Perú.

Majluf, A. 1993. Marginalidad, inteligencia y rendimiento escolar. Brandom Enterprise. Lima, Perú 274 pp.

Mejía, E.; Ayala, M; Zárate, M y G. Ruíz. 1995. Enterobiasis en niños del distrito de Moche en relación a algunos factores epidemiológicos. Res. II Cong. Per. Parasitología pp. (17).

Naupay, A., Gárate, I. Y Morante, Y. 1992. Enteroparasitosis y estado nutricional en niños de la comunidad de Santo Toribio de HuaylasAncash. Res. I Cong. Investigaciones en Ciencias de la Salud. UNMSM, I 23 pp.

Salomons, N. y Rosales, F. 1986. Parasitosis y Nutrición. Inst. de Nutrición de Centroamércia y Panamú. Cuaderno de Nutrición N. 3 : 3-8.

Solo, R. y Vega, L. 1993. Determinación de la influencia de la anemia y la deficiencia del huevo en el rendimiento escolar. Diagnóstico 24: 5-8. 